\title{
Relationship of preoperative NT-pro-BNP with clinical, perioperative and prognostic markers in cardiac surgery: Preliminary study results
}

Irina A. Akhmedova, Taalaibek Z. Kudaiberdiev, Damirbek A. Abibillaev, Akylbek A. Zhooshev, Dolonbek E. Zaripov, Kaiyrnisa T. Tilemanbetova, Guliza N. Naizabekova

Scientific-Research Institute of Heart Surgery and Organ Transplantation, Bishkek, Kyrgyzstan

\begin{abstract}
Objective: We aimed to define the relationship of preoperative N-terminal-pro-brain type natriuretic peptide (NTpro-BNP) testing in routine practice of cardiac surgery with clinical, perioperative variables, surgical outcomes and complications in pediatric and adult cardiac surgery patients. In addition, we assessed relationship of NT-pro-BNP with EuroSCORE II in adult patients undergoing cardiac surgery.

Methods: A total of 48 patients who underwent cardiac surgery in our institution were enrolled into this retrospective observational cohort study. According to age aspects pediatric ( $P G, n=20)$ and adult $(A G, n=28)$ group of patients were separately evaluated. Each group further was categorized into two subgroups (group 1 and 2 ) on the basis of cut-off points of NT-pro-BNP level ( 430 and $250 \mathrm{pg} / \mathrm{ml}$ in adults and children, respectively). Preoperative NT-pro- BNP was obtained from patients. Statistical tests were conducted to reveal differences in clinical and perioperative variables among NT-pro-BNP groups and relationship of the assay with baseline clinical and operative parameters, as well as postoperative outcomes.

Results: In adults undergoing cardiac surgery, statistically significant differences were found between groups with low and high levels of NT-pro-BNP by NYHA classes (I to III, p<0.001), body mass index (BMI) values (mean (SD) $31.7(3.15)$ vs 25.9 (3.8) kg/cm², p=0.017), estimated glomerular filtration rate (eGFR) (111.4 (26.4) vs 77.9 (22.4) $\left.\mathrm{ml} / \mathrm{min} / 1.73 \mathrm{~m}^{2}, \mathrm{p}=0.036\right)$, moderately impaired renal function $(20 \%$ vs $72.7 \%, \mathrm{p}=0.049)$, need for inotropes ( $28.6 \%$ vs $86.7 \%, p=0.006)$, and mean EuroSCORE II (0.81 (0.19) vs $1.7(0.5), p=0.008)$. There was the positive significant correlation of NT-pro-BNP levels with EuroSCORE II, NYHA class, need for inotropes, renal function and left ventricular (LV) function categories, while its negative correlation was found with BMI, LV ejection fraction and eGFR parameters. In PG - NT-pro-BNP was negatively correlated with age, body surface area, LV end-diastolic and endsystolic dimensions.

Conclusion: In adult patients undergoing cardiac surgery, preoperative NT-pro-BNP level correlated well with several baseline and clinical-operative parameters, including BMI, renal and LV function, NYHA class, need for inotropes support, and EuroSCORE II. Further studies are needed to define its exact diagnostic and prognostic significance in pediatric patients.
\end{abstract}

Key words: cardiac surgery procedures, congestive heart failure, NT-pro-BNP, correlation, prognosis, surgical risk, surgery outcomes, early postoperative complications, EuroSCORE, renal failure

(Heart Vessels Transplant 2020; 4; doi: 10.24969/hvt.2020.205)

\section{Introduction}

Heart failure may be a result of vast majority of cardiovascular disorders including myocardial damage, valve pathology, conduction and rhythm abnormalities, and pericardial diseases $(1,2)$. History of most of the patients reveals both cardiac and noncardiac comorbid conditions as the underlying factors of chronic heart failure (HF) (3). The incidence of $\mathrm{HF}$ is steadily increasing and remains the leading cause of hospitalization and in-hospital mortality in elderly population (age $\geq 65$ years) (4-6). By the improvement of current management, great successes have been achieved both in pharmacologic and surgical treatment of chronic HF. 
Heart surgeries are quite challenging procedures, which require a cardiac-pulmonary bypass (CPB), and other settings. Additionally, high risk of complications are observed in postoperative period. Advanced age of patients and huge number of comorbid issues lead to increase of disease severity requiring more complex operations including simultaneous, hybrid and multistage procedures $(7,8)$. Furthermore, despite the significant improvement of healthcare, surgical operations performed in children with congenital heart diseases (CHD) are accompanied by high morbidity and mortality both early and late postoperative periods. Implementation of the robust prognostic biomarkers besides the routine ones used in daily clinical practice is crucial in postoperative management of pediatric patients in intensive care unit (ICU).

Several multifactorial scores have been used for preoperative risk stratification and surgery outcomes. But, no single risk assessment tool for cardiac surgery includes preoperative measurement of brain-type natriuretic peptides (BNP) in spite of their accurate correlation with ventricular dysfunction and HF (10). On the other hand, some studies recommend cautious application of risk assessment models in cardiac surgery for different population groups, due to existence of great variety by gender, ethnicity and geographic factors (11).

BNP, a valuable marker of $\mathrm{HF}$ is the member of natriuretic peptides family, which is secreted into bloodstream in response to volume overload or conditions causing myocardial stretching of ventricles. Compared to atrial natriuretic peptides (ANP), BNP is synthesized not only by atria, but also by ventricles, particularly in patients with HF (12). Circulating concentrations of several cardiac natriuretic peptides, including ANP, BNP and their N-terminal prohormones (NT-pro-ANP and NT-pro-BNP) are elevated both in patients with $\mathrm{HF}$ signs and asymptomatic left ventricular (LV) dysfunction (13-15). In practical sense, BNP and NT-pro-BNP are interchangeable and can be used according to local preference and availability. For diagnostic workup of mild HF and subclinical LV dysfunction NT-pro-BNP is preferred over BNP (15).

Duceppe $E$ et al. proposed the prognostic role of NT-pro-BNP in adverse cardiovascular outcomes and mortality risk assessment of patients undergoing noncardiac surgery within postoperative thirty days (16). In cardiac surgery, preoperative levels of NT-pro-BNP well correlate with ventilation time, duration of intensive care stays and perioperative pharmacological inotropic support (17). According to Hutfless et al, the level of BNP $>385 \mathrm{pg} / \mathrm{ml}$ may predict postoperative complications and mortality within twelve months after the cardiac surgery (10).

The study of Liu $\mathrm{H}$. et al. revealed the level of NT-proBNP at $2773.5 \mathrm{pg} / \mathrm{ml}$ as a cut-off value (sensitivity $63.6 \%$ and specificity $80.8 \%$ ) for mortality prognosis within 30 days after surgery (18). However, the evidence of clinical and prognostic utility of NT-proBNP in cardiac surgery is still emerging.

In this preliminary study, we objected to define the relationship of preoperative NT-pro-BNP testing in routine practice of cardiac surgery with clinical, perioperative variables (cardiopulmonary bypass time, aortic cross-clamp time, ventilation duration, need for inotropes, duration of intensive care stays), surgical outcomes and complications in pediatric and adult cardiac surgery patients. In addition, we assessed relationship of NT-pro-BNP with EuroSCORE II in adult patients undergoing cardiac surgery.

\section{Methods}

\section{Study design}

Retrospective, observational cohort study.

\section{Study population}

A total of 48 patients enrolled into study underwent elective cardiac surgery in Scientific Research Institute of Heart Surgery and Organ Transplantation (SRIHSOT) within two months of 2019 (September-November). Pediatric patients constituted $42 \%(n=20)$ operated due to CHD, adults comprised 58\% (n=28) and underwent coronary artery bypass surgery (CABG), aortic (AVR) and mitral valve (MVR) replacement surgeries and myxoma resection surgery.

Study population was categorized into two groups by age aspects: pediatric (PG) and adult (AG) ones. Further each age group was divided into two subgroups, according to NT-pro-BNP levels: $1^{\text {st }}$ subgroup included cases with less than $430 \mathrm{pg} / \mathrm{ml}(\mathrm{AG})$ (19) and $250 \mathrm{pg} / \mathrm{ml}$ (PG) (20), 2nd subgroup more than those cut-off points.

The NYHA IV class patients were excluded, though they did not undergo surgery. Patients with altered renal function (chronic kidney disease IIIB-V) were also excluded. Due to absence of NT-pro-BNP data 4 pediatric and 6 adult patients were excluded from the study.

Informed consent for procedures was obtained from all adult patients and from parents (guardians) of children patients. 


\section{Data analysis}

We collected the following baseline demographic data from patient records: age (expressed in years (AG) and months (PG)), sex (male or female), body mass index (weight/height ${ }^{2}$ in $A G$ ), body surface area (by Haycock formula in $P G$ ), underlying diagnoses, type of surgical operations, presence of risk factors for adverse outcomes of CHF (presence or absence of the dyslipidemia/atherosclerosis, myocardial infarction (MI), arterial hypertension, diabetes mellitus (DM), preoperative cardiac arrhythmia).

For preoperative assessment of CHF in adults, we used New-York Heart Association functional classification (NYHA) proposed in 1964, while for pediatric patients - modified Ross classification for pediatric $\mathrm{HF}(21,22)$.

For baseline clinical data we collected serum creatinine levels, estimated glomerular filtration rate (eGFR) assessed by modified Cockroft-Gault formula (23), renal function categories (normal renal function $\left(\geq 85 \mathrm{ml} / \mathrm{min} / 1.73 \mathrm{~m}^{2}\right)$, moderately or severely impaired $\quad\left(85-45 \mathrm{ml} / \mathrm{min} / 1,73 \mathrm{~m}^{2}\right.$ and $<45$ $\mathrm{ml} / \mathrm{min} / 1,73 \mathrm{~m}^{2}$ ), echocardiographic LV indices (LV end-diastolic (LVEDD) and end-systolic (LVESD), dimensions, LV ejection fraction (EF) by Teichholz and Simpson methods) and LV systolic function categories (reduced $\mathrm{EF}<40 \%$, moderate (EF $40-50 \%$ ) or preserved $(\mathrm{EF} \geq 50 \%))$, Doppler-derived systolic pulmonary artery pressure (SPAP), pulmonary artery hypertension (PAH) categories (normal SPAP $(<30 \mathrm{mmHg})$, moderate PAH (31-55 mm $\mathrm{mg}$ ) and severe PAH (>55 $\mathrm{mmHg})$ ).

For perioperative data we collected $\mathrm{CPB}$ and aortic cross-clamp (ACCT) times, duration of ICU stay, ventilation time and need for pharmacological inotropes, bleeding event. Surgical risk was calculated according to EuroSCORE II by evaluating following factors: age, gender, eGFR, NYHA functional class, urgency and volume of the cardiac surgery, SPAP, LVEF, Canadian Cardiovascular Society functional class of angina, presence/absence of extracardiac arteriopathy, poor mobility, previous cardiac surgery, chronic obstructive pulmonary disease, active endocarditis, recent $\mathrm{MI}, \mathrm{DM}$ on insulin treatment, critical state, thoracic aorta surgery $(24,25)$.

For postoperative analysis surgery outcomes (alive vs died), complications (acute kidney injury (AKI), acute respiratory failure, chronic HF exacerbation) and arrhythmia (presence or absence) were used.
First sample was obtained in operation theatre prior to anesthesia. Second sampling was performed after 24 hours of operation in patients ventilated and intravenously supported by inotropes. Serum investigation was performed immediately after blood sampling in UPT - 3A analyzer, by electrochemiluminescence immunoassay (ECLIA) method, measurement units - pg/ml (Beijing Hotgen Biotech Co.LTD, China).

In adults, a $<300 \mathrm{pg} / \mathrm{ml}$ level of NT-pro-BNP could safely exclude likelihood of $\mathrm{HF}$ at any age. According to age aspects, for individuals aged 21-50 years $\geq 450 \mathrm{pg} / \mathrm{ml}$, $50-75$ years $\geq 900 \mathrm{pg} / \mathrm{ml}$, and elder 75 years $\geq 1800 \mathrm{pg} / \mathrm{ml}$ levels confirm HF. The values between $300 \mathrm{pg} / \mathrm{ml}$ and aforementioned levels remained as grey zone (26).

In pediatric patients to date, no clear standards and no normal ranges of NT-pro-BNP were established. Based on ROC curve some studies revealed the $598 \mathrm{ng} / \mathrm{l}$ as a cut-off level for HF diagnosis in children (27).

We used as cut-off values $430 \mathrm{pg} / \mathrm{ml}$ for adult cardiac surgery patients, as the higher values were associated with adverse outcomes after cardiac surgery in adults (19) and $250 \mathrm{pg} / \mathrm{ml}$ for pediatric cardiac surgery patients (20).

\section{Statistical analysis}

Statistical analysis was performed using SPSS for Windows software (IBM, New York, USA).

Continuous variables are depicted in mean (standard deviation, SD), median (min-max range), categorical variables as counts (percentage).

Chi-square test was used to assess differences for categorical variables, and Mann Whitney $U$ test - for continuous variables. Nonparametric Spearman correlation analysis was used to assess correlation of NT-pro-BNP level with baseline and clinicalperioperative variables. $P$ value of less than 0.05 was accepted as significant value determination $(p<0.05)$. Due to scarcity of data and small sample size we used only descriptive statistics for pediatric groups and Spearman correlation analysis of relationship of NTpro-BNP with clinical and perioperative variables. 


\section{Results}

\section{Adult cardiac surgery patients}

Baseline population demographics

Patient population was categorized into two groups on the basis of preoperative NT-pro-BNP level. A $430 \mathrm{pg} / \mathrm{ml}$ was accepted as the cutoff value for grouping in AG. Baseline clinical variables and their statistical results are highlighted in Table 1 . Mean age was 60.01 years. Age range: 34 to 72 years. All operations were performed in circumstance of CPB except the stent-graft implantation: CABG, AVR, MVR, $A V R$ and MVR, left atrial (LA) myxoma resection, and stent-graft implantation.

According to age, sex, main diagnosis, surgery type, risk factors of $\mathrm{HF}$ and postoperative clinical outcomes no significant differences were found among groups. Significant results were obtained for BMI ranges and categorizations (slim $\left(<25 \mathrm{~kg} / \mathrm{m}^{2}\right)$ and overweight or obese $\left.\left(\geq 25 \mathrm{~kg} / \mathrm{m}^{2}\right)\right)$ : $\mathrm{p}$-value was 0.03 and 0.017 , respectively. More patients that are obese were in group of low NT-pro-BNP level. There were significantly more patients with higher NYHA class in group of patients with high NT-pro-BNP levels $(p<0.001)$, especially more patients with class III $(p=0.03)$.

\section{Clinical and operative variables}

All results are depicted in Table 2. Echocardiographic data regarding cardiac chamber values, LV systolic function and estimated SPAP did not show significant differences $(p>0.05)$. Similar calculations were obtained for creatinine level, intraoperative and ICU parameters, namely, ventilation time (VT), CPB, ACCT, and duration of ICU stay. Nevertheless, significant differences were observed among groups by estimated glomerular filtration rate (EGFR, $p=0.036)$, frequency of renal function impairment (moderately impaired, $p=0.049$ ), need for inotropes in early postoperative period $(p=0.006)$ and EuroSCORE
II scale $(p=0.008)$. Patients with high NT-pro-BNP levels had markedly lower eGFR rate $(p=0.036)$, higher number of patients with renal function impairment $(p=0.049)$, and more patients in this group needed inotropic support $(p=0.006)$. The patients with higher NT-pro-BNP had higher surgical risk values compared to subjects with lower levels of NT-pro-BNP $(p=0.008)$. However, there were no differences in adverse outcomes and complications between groups. We also did not observe any bleeding events.

Correlations of NT-pro-BNP with clinical and perioperative variables (Table 3)

NT-pro-BNP levels positively, strongly and significantly correlated with EuroSCORE II, NYHA classes and eGFR ( $p=0.001)$ with rho-values $0.73,0.87$ and -0.73 , respectively. Significant results $(p<0.05)$ were also found for the following parameters with corresponding rho-values: need for inotropes (0.62), renal function categories (0.72), EF obtained by Teichholz method (-0.47) and LV function categories (0.52).

NT-pro-BNP was negatively correlated with BMI ranges (-0.73), meaning higher BNP levels in lower BMI values. But higher NT-pro-BNP levels were related to lower values of EF (-0.47), and NT-pro-BNP levels were positively correlated to systolic indices (categories $<40 \%$ and $<50 \%$ of EF) (rho $0.52, p=0.01$ ).

\section{Pediatric cardiac surgery patients}

\section{Baseline demographic indices}

According to cut-off point of NT-pro-BNP level PG was grouped into less or more than $250 \mathrm{pg} / \mathrm{ml}$. As can be seen from tables 4 and 5 , only descriptive statistics of baseline clinical and operative parameters were highlighted. The mean age was $32.1 \pm 30.3$ months, median: 23 months, age range 14 days (0 months)-96 months (8 years). 


\begin{tabular}{|c|c|c|c|}
\hline Variable & $\begin{array}{l}\text { Group } 1 \\
\text { NT-pro-BNP }<430, \mathrm{pg} / \mathrm{ml}\end{array}$ & $\begin{array}{l}\text { Group } 2 \\
\text { NT-pro-BNP>430, pg/ml }\end{array}$ & p \\
\hline $\begin{array}{l}\text { Age, years } \\
\text { Mean (SD) } \\
\text { Median (range) }\end{array}$ & $\begin{array}{l}58.0(12.17) \\
60(34-69)\end{array}$ & $\begin{array}{l}61.46(6.32) \\
62(48-72)\end{array}$ & 0.672 \\
\hline $\begin{array}{l}\text { Sex, } \mathbf{n}(\%) \\
\text { Male } \\
\text { Female }\end{array}$ & $\begin{array}{l}5(71.4) \\
2(28.6)\end{array}$ & $\begin{array}{l}9(60.0) \\
6(40.0)\end{array}$ & 0.604 \\
\hline $\begin{array}{l}\text { Surgery, } \mathbf{n}(\%) \\
\text { CABG } \\
\text { MVR } \\
\text { AVR } \\
\text { Myxoma resection } \\
\text { AVR+MVR }\end{array}$ & $\begin{array}{l}4(57.1) \\
1(14.3) \\
1(14.3) \\
0 \\
1(14.3)\end{array}$ & $\begin{array}{l}8(53.3) \\
1(6.7) \\
3(20) \\
1(6.7) \\
2(13.3)\end{array}$ & 0.928 \\
\hline $\begin{array}{l}\text { Diagnosis, } \mathbf{n}(\%) \\
\text { CAD } \\
\text { RVHD } \\
\text { BiAV } \\
\text { Myxoma } \\
\text { IE }\end{array}$ & $\begin{array}{l}4(57.1) \\
1(14.3) \\
1(14.3) \\
0 \\
1(14.3)\end{array}$ & $\begin{array}{l}7(46.7) \\
6(40) \\
1(6.7) \\
1(6.7) \\
0\end{array}$ & 0.405 \\
\hline $\begin{array}{l}\text { NYHA class, } \mathbf{n}(\%) \\
\text { I } \\
\text { II } \\
\text { III }\end{array}$ & $\begin{array}{l}5(71.4) \\
2(28.6) \\
0\end{array}$ & $\begin{array}{l}0 \\
5(33.3) \\
10(66.7)\end{array}$ & $<0.001$ \\
\hline $\begin{array}{l}\text { BMI, } \mathbf{k g} / \mathbf{c m}^{2} \\
\text { Mean (SD) } \\
\text { Median (range) }\end{array}$ & $\begin{array}{l}31.7(3.15) \\
30.7(28.6-36.5)\end{array}$ & $\begin{array}{l}25.9(3.8) \\
25.5(19.5-32.5)\end{array}$ & 0.017 \\
\hline $\begin{array}{l}\text { BMI categories, } \mathbf{n}(\%) \\
<25 \\
25-30 \\
>30\end{array}$ & $\begin{array}{l}0 \\
2(40) \\
3(60)\end{array}$ & $\begin{array}{l}5(50) \\
4(40) \\
1(10)\end{array}$ & 0.06 \\
\hline Dyslipidemia n (\%) & $3(42.9)$ & $9(64.3)$ & 0.35 \\
\hline Myocardial infarction, $\mathbf{n}(\%)$ & $1(14.3)$ & $5(35.7)$ & 0.306 \\
\hline Hypertension, $\mathrm{n}(\%)$ & $4(57.1)$ & $8(66.7)$ & 0.678 \\
\hline Diabetes mellitus, $\mathrm{n}(\%)$ & $6(85.7)$ & $11(91.7)$ & 0.683 \\
\hline Preoperative Arrhythmia, n (\%) & 0 & $3(23.1)$ & 0.23 \\
\hline Postoperative Arrhythmia, n (\%) & 0 & $8(61.5)$ & 0.03 \\
\hline $\begin{array}{l}\text { Outcome, } \mathbf{n}(\%) \\
\text { Alive } \\
\text { Died }\end{array}$ & $\begin{array}{l}6(85.7) \\
1(14.3)\end{array}$ & $\begin{array}{l}12(80) \\
3(20)\end{array}$ & 0.746 \\
\hline $\begin{array}{l}\text { Complications, } \mathbf{n} \text { (\%) } \\
\text { AKI } \\
\text { AKI+CHFe } \\
\text { CHFe } \\
\text { Resp.F+CHFe } \\
\text { none }\end{array}$ & $\begin{array}{l}0 \\
0 \\
1(14.3) \\
0 \\
6(85.7)\end{array}$ & $\begin{array}{l}1(6.7) \\
1(6.7) \\
0 \\
1(6.7) \\
12(80)\end{array}$ & 0.469 \\
\hline
\end{tabular}




\begin{tabular}{|c|c|c|c|}
\hline Variable & $\begin{array}{c}\text { Group } 1 \\
\text { NT-pro-BNP }<430 \mathrm{pg} / \mathrm{ml}\end{array}$ & $\begin{array}{c}\text { Group } 2 \\
\text { NT-pro-BNP>430 pg/ml }\end{array}$ & $p$ \\
\hline $\begin{array}{l}\text { LVESD, } \mathbf{m m} \\
\text { Mean (SD) } \\
\text { Median (range) }\end{array}$ & $\begin{array}{l}37.2(10.6) \\
33(30-60)\end{array}$ & $\begin{array}{l}38.6(7.6) \\
39(29-52)\end{array}$ & 0.501 \\
\hline $\begin{array}{l}\text { LVEDD, } \mathbf{~ m m} \\
\text { Mean (SD) } \\
\text { Median (range) }\end{array}$ & $\begin{array}{l}54.4(5.6) \\
55(43-60)\end{array}$ & $\begin{array}{l}53.9(5.8) \\
52(46-63)\end{array}$ & 0.626 \\
\hline $\begin{array}{l}\text { EF Teichholz, \% } \\
\text { Mean (SD) } \\
\text { Median (range) }\end{array}$ & $\begin{array}{l}63.4(5.3) \\
63(57-70)\end{array}$ & $\begin{array}{l}56.6(10.01) \\
59(34-69)\end{array}$ & 0.156 \\
\hline $\begin{array}{l}\text { LV function, } \mathbf{n}(\%) \\
\text { reduced } \mathrm{EF}<40 \% \\
\text { moderate reduced EF } 40-50 \% \\
\text { preserved } \mathrm{EF}>50 \%\end{array}$ & $\begin{array}{l}0 \\
0 \\
7(100)\end{array}$ & $\begin{array}{l}1(7.1) \\
4(28.6) \\
9(64.3)\end{array}$ & 0.194 \\
\hline $\begin{array}{l}\text { EF Simpson, \% } \\
\text { Mean (SD) } \\
\text { Median (range) }\end{array}$ & $\begin{array}{l}54.5(4.13) \\
54.5(49-60)\end{array}$ & $\begin{array}{l}51.8(6.3) \\
54(43-59)\end{array}$ & 0.454 \\
\hline $\begin{array}{l}\text { Creatinine, } \boldsymbol{\mu m o l} / \mathbf{I} \\
\text { Mean (SD) } \\
\text { Median (range) }\end{array}$ & $\begin{array}{l}85.3(19.4) \\
85.3(56.1-115.4)\end{array}$ & $\begin{array}{l}92.06(21.9) \\
86.3(66.2-149.4)\end{array}$ & 0.794 \\
\hline $\begin{array}{l}\text { EGFR, } \mathrm{ml} / \mathrm{min} / \mathbf{1 . 7 3 \mathrm { m } ^ { 2 }} \\
\text { Mean (SD) } \\
\text { Median (range) }\end{array}$ & $\begin{array}{l}111.4(26.4) \\
119.7(78.8-137.7)\end{array}$ & $\begin{array}{l}77.9(22.4) \\
75.5(55.4-132)\end{array}$ & 0.036 \\
\hline $\begin{array}{l}\text { RF, } \mathbf{n}(\%) \\
\text { Normal }\left(>85 \mathrm{ml} / \mathrm{min} / 1.73 \mathrm{~m}^{2}\right) \\
\text { Moderate impairment }\left(60-45 \mathrm{ml} / \mathrm{min} / 1.73 \mathrm{~m}^{2}\right)\end{array}$ & $\begin{array}{l}4(80) \\
1(20)\end{array}$ & $\begin{array}{l}3(27.3) \\
8(72.7)\end{array}$ & 0.049 \\
\hline $\begin{array}{l}\text { SPAP, } \mathbf{m m ~ H g} \\
\text { Mean (SD) } \\
\text { Median (range) }\end{array}$ & $\begin{array}{l}34.6(5.5) \\
35(28-40)\end{array}$ & $\begin{array}{l}39(5.1) \\
40(30-50)\end{array}$ & 0.187 \\
\hline $\begin{array}{l}\text { PAH, } \mathbf{n}(\%) \\
\text { No }(<30 \mathrm{mmHg}) \\
\text { Moderate PAH }(31-55 \mathrm{mmHg})\end{array}$ & $\begin{array}{l}2(40) \\
3(60)\end{array}$ & $\begin{array}{l}1(8.3) \\
11(91.7)\end{array}$ & 0.119 \\
\hline $\begin{array}{l}\text { ICU duration, days } \\
\text { Mean (SD) } \\
\text { Median (range) }\end{array}$ & $\begin{array}{l}3.8(4.5) \\
1.0(1.0-1.13)\end{array}$ & $\begin{array}{l}2.6(2.9) \\
1.0(1.0-1.10)\end{array}$ & 0.817 \\
\hline $\begin{array}{l}\text { CPB time, min } \\
\text { Mean (SD) } \\
\text { Median (range) }\end{array}$ & $\begin{array}{l}124.0(93.7) \\
84.5(64.0-263.0)\end{array}$ & $\begin{array}{l}108.3(58.6) \\
103.0(40.0-219.0)\end{array}$ & 0.938 \\
\hline $\begin{array}{l}\text { ACCT, } \min \\
\text { Mean (SD) } \\
\text { Median (range) }\end{array}$ & $\begin{array}{l}88.2(66.5) \\
56.5(52.0-188.0)\end{array}$ & $\begin{array}{l}74.3(50.3) \\
65.0(29.0-180.0)\end{array}$ & 0.643 \\
\hline Inotropes need, $\mathrm{n}(\%)$ & $2(28.6)$ & $13(86.7)$ & 0.006 \\
\hline $\begin{array}{l}\text { Ventilation time, hours } \\
\text { Mean (SD) } \\
\text { Median (range) }\end{array}$ & $\begin{array}{l}38.5(62.3) \\
8.0(6.0-132.0)\end{array}$ & $\begin{array}{l}9.7(4.4) \\
8.0(4.0-19.0)\end{array}$ & 0.84 \\
\hline $\begin{array}{l}\text { EuroSCORE II } \\
\text { Mean (SD) } \\
\text { Median (range) }\end{array}$ & $\begin{array}{l}0.81(0.19) \\
0.75(0.64-1.13)\end{array}$ & $\begin{array}{l}1.7(0.5) \\
1.83(0.67-2.2)\end{array}$ & 0.008 \\
\hline
\end{tabular}




\begin{tabular}{l|l|l|}
\hline \multicolumn{3}{|l|}{ Table 3. Correlation of NT-pro-BNP with baseline, clinical and operative variables in adult group } \\
\hline Variable & Rho & p \\
\hline EuroSCORE II & 0.73 & 0.001 \\
\hline NYHA class & 0.87 & $<0.001$ \\
\hline Inotropes need & 0.62 & 0.002 \\
\hline BMI & -0.73 & 0.002 \\
\hline EF Teichholz & -0.47 & 0.02 \\
\hline LV function & 0.52 & 0.01 \\
\hline EGFR & -0.73 & 0.001 \\
\hline Renal function & 0.72 & 0.02 \\
\hline $\begin{array}{l}\text { BMI - body mass index, EGFR-estimated glomerular filtration rate, EF - ejection fraction, LV - left } \\
\text { ventricle, NT-pro-BNP-N-terminal }\end{array}$ & pro brain type natriuretic peptide, NYHA class-New-York Heart \\
\hline
\end{tabular}

Of 16 patients 9 are males (45\%).The average NT-proBNP level was $3602.5(9094) \mathrm{pg} / \mathrm{ml}$, $\min 217.9 \mathrm{pg} / \mathrm{ml}$, $\max 35000 \mathrm{pg} / \mathrm{ml}$. Mean body surface area (BSA): 0.48 $\left(0.21 \mathrm{~m}^{2}\right)$, median: $0.45 \mathrm{~m}^{2}$ range: $0,21-0,91 \mathrm{~m}^{2}$. Patients were distributed according to underlying $\mathrm{CHD}$ as follows: ventricular septal defect (VSD, $n=6)$, atrial septal defect (ASD, $n=2), A S D+V S D(n=1), A S D+$ partial anomalous pulmonary venous return (PAPVR) $(n=1)$, patent ductus arteriosus (PDA, $n=1$ ), Fallot tetralogy (TOF, $n=2$ ), complete atrioventricular septal defect (AVSDc, $n=1)$, coarctation of aorta (CoA, $n=1)$, pulmonary stenosis ( $P S, n=1$ ). Performed surgical interventions with use of CPB were as follows: ASD closure $(n=2), V S D$ correction $(n=6), V S D+A S D$ closure $(n=1)$, ASD closure and PAPVR repair $(n=1)$, complete $\operatorname{AVSD}$ correction $(n=1)$, radical correction of TOF $(n=1)$, pulmonary valvulotomy $(n=1)$. Surgical procedures without CPB were systemic-pulmonary shunting in TOF ( $n=1)$, PDA ligation ( $n=1)$, and CoA correction $(n=1)$. According to NYHA Ross classification children were distributed by: I ( $n=3), I I(n=4)$ and III ( $n=9)$.
Clinical and operative variables (Table 5)

Echocardiographic indices: LVEDD: 29.07 (7.08) mm, LVESD: 17.28 (4.7) mm, LVEF: 72.1 (4.4)\%. Systolic pulmonary artery pressure (SPAP): 56.8 (17.1)mmHg. Creatinine level: 38.2 (14.9) $\mu \mathrm{mol} / \mathrm{I}$. CPB time: 50.2 (21.4)min, ACCT: 26.0 (11.5) $\mathrm{min}$, ICU duration: 2.07(1.6) days, ventilation time: 17.7 (34.8) h, need for inotropic support: 12 (75\%). Only single lethal case was observed during hospital stay due to exacerbation of CHF (0.62\%).

\section{Correlations}

A non-parametric correlation represented statistically significant correlation of NT-pro-BNP with following variables: age $(p=0.038)$, BSA $(p=0.002)$, left ventricular diastolic $(p=0.024)$ and systolic diameters $(p=0.053)$ (all are negatively correlated) (Table 6). 
Table 4. Baseline clinical characteristics of pediatric patients

\begin{tabular}{|c|c|c|c|}
\hline Variable & $\begin{array}{l}\text { Group } 1 \\
\text { NT-pro-BNP<250, pg/ml }\end{array}$ & $\begin{array}{l}\text { Group } 2 \\
\text { NT-pro-BNP>250, pg/ml }\end{array}$ & Number of cases \\
\hline $\begin{array}{l}\text { Age, months } \\
\text { Mean (SD) } \\
\text { Median (range) }\end{array}$ & $\begin{array}{l}52.0(27.7) \\
36(36-84)\end{array}$ & $\begin{array}{l}26.7(29.9) \\
11(1-96)\end{array}$ & 14 \\
\hline $\begin{array}{l}\text { Sex, } \mathbf{n}(\%) \\
\text { Male } \\
\text { Female }\end{array}$ & $\begin{array}{l}1(33.3) \\
2(66.7)\end{array}$ & $\begin{array}{l}6(46.2) \\
7(53.8)\end{array}$ & 16 \\
\hline $\begin{array}{l}\text { Surgery, } \mathbf{n} \text { (\%) } \\
\text { ASD closure } \\
\text { VSD closure } \\
\text { ASD+VSD correction } \\
\text { PDA ligation } \\
\text { AVSDc correction } \\
\text { TOF radical correction } \\
\text { MBTS } \\
\text { CoA correction } \\
\text { PV surgery }\end{array}$ & $3(100)$ & $\begin{array}{l}2(15.3) \\
3(23.07) \\
1(7.6) \\
1(7.6) \\
1(7.6) \\
1(7.6) \\
1(7.6) \\
1(7.6) \\
1(7.6)\end{array}$ & 16 \\
\hline $\begin{array}{l}\text { Diagnosis, } \mathbf{n} \text { (\%) } \\
\text { ASD } \\
\text { VSD } \\
\text { ASD+VSD } \\
\text { PDA } \\
\text { ASD+PAPVR } \\
\text { AVSDc } \\
\text { PS } \\
\text { TOF } \\
\text { CoA }\end{array}$ & $3(100)$ & $\begin{array}{l}2(15.3) \\
3(23.07) \\
1(7.6) \\
1(7.6) \\
1(7.6) \\
1(7.6) \\
1(7.6) \\
2(15.3) \\
1(7.6)\end{array}$ & 16 \\
\hline $\begin{array}{l}\text { NYHA Ross, n (\%) } \\
\text { I } \\
\text { II } \\
\text { III }\end{array}$ & $\begin{array}{l}1(33.3) \\
1(33.3) \\
1(33.3)\end{array}$ & $\begin{array}{l}2(15.3) \\
3(23.07) \\
8(61.5)\end{array}$ & 16 \\
\hline $\begin{array}{l}\text { BSA, } \mathbf{m} \mathbf{2} \\
\text { Mean (SD) } \\
\text { Median (range) }\end{array}$ & $\begin{array}{l}0.7(0.17) \\
0.65(0.59-0.91)\end{array}$ & $\begin{array}{l}0.42(0.18) \\
0.37(0.21-0.80)\end{array}$ & 14 \\
\hline $\begin{array}{l}\text { Outcome, } \mathbf{n}(\%) \\
\text { Alive } \\
\text { Died }\end{array}$ & $\begin{array}{l}3(100) \\
0\end{array}$ & $\begin{array}{l}12(92.3) \\
1(7.6)\end{array}$ & 16 \\
\hline $\begin{array}{l}\text { Complications, } \mathbf{n}(\%) \\
\text { CHFe } \\
\text { None }\end{array}$ & $\begin{array}{l}0 \\
3(100)\end{array}$ & $\begin{array}{l}1(7.6) \\
12(92.3)\end{array}$ & 16 \\
\hline
\end{tabular}




\begin{tabular}{|c|c|c|c|}
\hline Variable & $\begin{array}{l}\text { Group } 1 \\
\text { NT-pro-BNP<250 pg/ml }\end{array}$ & $\begin{array}{l}\text { Group } 2 \\
\text { NT-pro-BNP>250 } \\
\mathrm{pg} / \mathrm{ml}\end{array}$ & Number of cases \\
\hline $\begin{array}{l}\text { LVESD, } \mathbf{m m} \\
\text { Mean (SD) } \\
\text { Median (range) }\end{array}$ & $\begin{array}{l}20.0(2.6) \\
19(18-23)\end{array}$ & $\begin{array}{l}16.5(4.9) \\
16(10-25)\end{array}$ & 14 \\
\hline $\begin{array}{l}\text { LVEDD, } \mathbf{~ m m} \\
\text { Mean (SD) } \\
\text { Median (range) }\end{array}$ & $\begin{array}{l}33.6(5.5) \\
34(28-39)\end{array}$ & $\begin{array}{l}27.8(7.1) \\
28(16-41)\end{array}$ & 14 \\
\hline $\begin{array}{l}\text { EF Teichholz, \% } \\
\text { Mean (SD) } \\
\text { Median (range) }\end{array}$ & $\begin{array}{l}71.0(5.5) \\
72(65-76)\end{array}$ & $\begin{array}{l}72.4(4.3) \\
73(64-78)\end{array}$ & 14 \\
\hline $\begin{array}{l}\text { Creatinine, } \boldsymbol{\mu m o l} / \mathbf{l} \\
\text { Mean (SD) } \\
\text { Median (range) }\end{array}$ & $\begin{array}{l}47.5(14.4) \\
49.4(32.2-60.9)\end{array}$ & $\begin{array}{l}35.7(14.7) \\
37.1(17.6-58.6)\end{array}$ & 14 \\
\hline $\begin{array}{l}\text { SPAP, } \mathbf{m m ~ H g} \\
\text { Mean (SD) } \\
\text { Median (range) }\end{array}$ & $\begin{array}{l}47.5(3.5) \\
47.5(45-50)\end{array}$ & $\begin{array}{l}59.1(18.6) \\
57.5(30-85)\end{array}$ & 10 \\
\hline $\begin{array}{l}\text { ICU duration, days } \\
\text { Mean (SD) } \\
\text { Median (range) }\end{array}$ & $\begin{array}{l}1.3(0.57) \\
1(1.0-2.0)\end{array}$ & $\begin{array}{l}2.2(1.7) \\
1.0(1.0-1.6)\end{array}$ & 14 \\
\hline $\begin{array}{l}\text { CPB time, min } \\
\text { Mean (SD) } \\
\text { Median (range) }\end{array}$ & $\begin{array}{l}64.3(28.5) \\
75.0(32.0-86.0)\end{array}$ & $\begin{array}{l}45.0(17.5) \\
45.0(19.0-73.0)\end{array}$ & 11 \\
\hline $\begin{array}{l}\text { ACCT, } \min \\
\text { Mean (SD) } \\
\text { Median (range) }\end{array}$ & $\begin{array}{l}32.6(18.4) \\
28.0(17.0-53.0)\end{array}$ & $\begin{array}{l}23.5(8.1) \\
24.0(11.0-34.0)\end{array}$ & 11 \\
\hline Inotropes need, n (\%) & $1(33.3)$ & $11(84.6)$ & 16 \\
\hline $\begin{array}{l}\text { Ventilation time, hours } \\
\text { Mean (SD) } \\
\text { Median (range) }\end{array}$ & $\begin{array}{l}7.6(1.5) \\
8.0(6.0-9.0)\end{array}$ & $\begin{array}{l}20.5(39.2) \\
8.0(2.0-138.0)\end{array}$ & 11 \\
\hline \multicolumn{4}{|c|}{$\begin{array}{l}\text { ACCT-aortic cross-clamp time, CPB-cardiac-pulmonary bypass, EF-left ventricular ejection fraction, } \\
\text { ICU-intensive care unit, LVEDD-left ventricular end-diastolic diameter, LVESD-left ventricular end- } \\
\text { systolic diameter, NT-pro-BNP-N-terminal pro brain type natriuretic peptide, SPAP-systolic pulmonary } \\
\text { artery pressure }\end{array}$} \\
\hline
\end{tabular}

Table 6. Correlation of NTpro-BNP with clinical and operative variables in pediatric patients

\begin{tabular}{|l|l|l|}
\hline Variable & rho & $\mathbf{p}$ \\
\hline Age & -0.55 & 0.038 \\
\hline BSA & -0.75 & 0.002 \\
\hline LV EDD & -0.59 & 0.024 \\
\hline LV ESD & -0.52 & 0.053 \\
\hline
\end{tabular}

BSA-body surface area, LVEDD-left ventricular end-diastolic diameter, LVESD-left ventricular endsystolic diameter, NT-pro-BNP-N-terminal pro brain type natriuretic peptide 


\section{Discussion}

Preliminary results of our study in adult patients who underwent cardiac surgery demonstrated that preoperative NT-pro-BNP levels are elevated in patients with advanced NYHA classes, LV dysfunction, higher surgical risk, worsened kidney function and need of inotropic support. In addition, a significant positive correlation was found between NT-pro-BNP level and EuroSCORE II, a prognostic scoring index which defines the complexity of surgery and patients' cardiac surgical risk; renal function categories, whether normal or moderately impaired; CHF NYHA functional class grading, from mild symptoms to limited daily activities; systolic function categories on the basis of LVEF; as well as need for inotropic support in early postoperative period. A negative correlation was observed by BMI, EF and GFR levels. However, we did not observe difference in surgery outcomes due to presumably small sample size.

Previous studies revealed the good correlation of LV global systolic dysfunction and symptom severity with NT-pro-BNP by means of LVEF with cut-off level of $50 \%$ (28). Nevertheless, recent findings advocate the relationships of BNP molecules with novel indices of global systolic function.

Several studies including large trials represented negative correlations between natriuretic peptides and BMI. According to these studies low NT-pro-BNP levels were detected both in overweight $(B M I=25-$ $\left.30 \mathrm{~kg} / \mathrm{m}^{2}\right)$ and obese $\left(\mathrm{BMI} \geq 30 \mathrm{~kg} / \mathrm{m}^{2}\right)$ subjects. Furthermore, these investigations defined similar levels of NT-pro-BNP levels as the cutoffs, in PRIDEstudy which included 204 subjects it was chosen $900 \mathrm{pg} / \mathrm{ml}$, in another trial with 1103 study population it was considered as $986 \mathrm{ng} / \mathrm{l}(29,30)$. Recent evidence supports the involvement of posttranslational processing of pro-BNP molecules, specifically the augmented glycosylation of threonine in $71^{\text {st }}$ position of pro-BNP and consequent decreasing in concentration of NT-pro-BNP molecules in overweight and obese patients (31). Despite the small sample size $(n=28)$ our patients also represented negative correlation with statistically significant results (rho=0.73, $p=0.002$ ) as in aforementioned literature reviews.

A univariate analysis showed that renal dysfunction was closely correlated with NT-pro-BNP level in assessment of the 30-day mortality (18). According to Su et al, NT-pro-BNP was found as an independent risk factor for development of acute kidney injury requiring renal replacement therapy in early postoperative period of cardiac surgery patients (32). Both continuous (eGFR) and categorical (renal impairment) variables in our study showed significant results among adult subgroups, as well as significant negative correlation of eGFR and positive correlation with renal impairment severity with NT-pro-BNP level, thus confirming previous studies.

Controversial findings exist in literature regarding to correlation of NT-pro-BNP and inotropic support in early postoperative period. According to Oztekin et al., no relationship was found between NT-pro-BNP level and need for inotropic support in patients who underwent isolated CABG surgery (33). In pediatric cardiac surgery, perioperative NT-pro-BNP accurately determines the prognosis of postoperative outcome including duration of mechanical ventilation, ICU stay and inotropic therapy (34).

Recent findings revealed the reliability and simplicity of preoperative NT-pro-BNP in predicting cardiac surgery risk or assisting the EuroSCORE II grading system $(35,36)$. Though in another study NT-pro-BNP and Euroscore II had both good predictive value for 3year mortality, no relation between them was reported (37). Our results extended those studies showing strong positive correlation of NT-pro-BNP with EuroSCORE II (rho 0.73, p=0.001). Further studies are needed to explore the additive predictive value of NT-pro-BNP and EuroSCORE II.

\section{Study limitations}

The main limitations of this preliminary study are small sample size and retrospective design. Results will be validated and reported in a further larger sample size study.

\section{Conclusion}

Preoperative NT-pro-BNP level in our cohort of adult patients is related to higher NYHA class, LV dysfunction, renal impairment and consequently higher requirement for inotropic support after surgery and is related with prognostic indicator as EuroSCORE II independently of type of surgery. There is a negative relation with BMI. Further study is needed to support our findings in adult and pediatric patients.

Peer-review: External and internal Conflict of interest: None to declare Authorship: I.A.A., T.Z.K., D.A.A., A.A.Zh., D.E.Z., K.T.T., G.N.N. are equally contributed the study and preparation of article Acknowledgments and funding: None to declare 


\section{References}

1. Mareev VY, Fomin IV, Ageev FT, Begrambekova YL, Vasyuk YA, Garganeeva AA, Gendlin GE, et al. Russian Heart Failure Society, Russian Society of Cardiology. Russian Scientific Medical Society of Internal Medicine Guidelines for Heart failure: chronic (CHF) and acute decompensated (ADHF). Diagnosis, prevention and treatment. Kardiologiia 2018; 58: 8-158.

2. Mareev VY, Ageev FT, Arutyunov GP, Korolev AV, Revishvili AS. Diagnosis and treatment of chronic heart failure. Working group on guideline preparation 2010.

3. Ponikowski $\mathrm{P}$, Voors AA, Anker SD, Bueno $\mathrm{H}$, Cleland JGF, Coats AJS, et al. 2016 ESC guidelines for the diagnosis and treatment of acute and chronic heart failure. The Task Force for the diagnosis and treatment of acute and chronic heart failure of the European Society of Cardiology (ESC). Developed with the special contribution of the Heart Failure Association (HFA) of the ESC. Eur Heart J 2016; 37; 2129-200.

4.Hunt SA, Abraham WT, Chin MH, Feldman AM, Francis GS, Ganiats TG, et al. American College of Cardiology; American Heart Association Task Force on Practice Guidelines; American College of Chest Physicians; International Society for Heart and Lung Transplantation; Heart Rhythm Society. ACC/AHA 2005 Guideline Update for the Diagnosis and Management of Chronic Heart Failure in the Adult: a report of the American College of Cardiology/American Heart Association Task Force on Practice Guidelines (Writing Committee to Update the 2001 Guidelines for the Evaluation and Management of Heart Failure): developed in collaboration with the American College of Chest Physicians and the International Society for Heart and Lung Transplantation: endorsed by the Heart Rhythm Society. Circulation 2005; 112: e154-235. DOI: 10.1161/CIRCULATIONAHA.105.167586

5. Lindenfeld J, Albert NM, Boehmer JP, Collins SP, Ezekowitz JA, Givertz MM, et al. HFSA 2010 comprehensive heart failure practice guideline. J Card Fail 2010; 16: e1-194. doi: 10.1016/j.cardfail.2010.04.004.

6. Bayés-Genís A. The circulating NT-pro-BNP level, a new biomarker for the diagnosis of heart failure in patients with acute shortness of breath). Rev Esp Cardiol 2005; 58: 1142-4.

7. Yaffee DW, Williams MR. Cardiovascular surgery in the elderly. Semin Thorac Cardiovasc Surg 2016; 28: 741-7. doi:10.1053/j.semtcvs.2016.08.007
8. Parissis H. Cardiac surgery: what the future holds? J Cardiothorac Surg 2011; 6: 93. doi:10.1186/1749-8090-6-93.

9. $Q u$ J, Liang $H$, Zhou $N$, Lijuan L, Wang $Y$, Jianbin L, et al. Perioperative NT-pro-BNP level: Potential prognostic markers in children undergoing congenital heart disease surgery. J Thorac Cardiovasc Surg 2017; 154: 631-40. doi:10.1016/j.jtcvs.2016.12.056.

10. Hutfless $R$, Kazanegra $R$, Madani $M$, Bhalla MA, Tata AT, Chen A, et al. Utility of B-type natriuretic peptide in predicting postoperative complications and outcomes in patients undergoing heart surgery. Journal of the American College of Cardiology 2004; 43: 1873-9. https://doi.org/10.1016/j.jacc.2003.12.048

11. Soo-Hoo S, Nemeth S, Baser O, Argenziano M1, Kurlansky P; East meets West: the influence of racial, ethnic and cultural risk factors on cardiac surgical risk model performance. Heart Asia 2018; 10: e010995. doi: 10.1136/heartasia-2017-010995.

12. Yasue $H$, Yoshimura $M$, Sumida $H$, Kikuta $K$, Kugiyama $K$, Jougasaki $M$, et al. Localization and mechanism of secretion of B-type natriuretic peptide in comparison with those of A-type natriuretic peptide in normal subjects and patients with heart failure. Circulation 1994; 90: 195-203.

13. Cowie MR, Struthers AD, Wood DA, Coats AJ, Thompson SG, Poole-Wilson PA, et al. Value of natriuretic peptides in assessment of patients with possible new heart failure in primary care. Lancet 1997; 350: 1349-53.

14. McDonagh TA, Robb SD, Murdoch DR, Morton JJ, Ford I, Morrison CE, et al. Biochemical detection of left-ventricular systolic dysfunction. Lancet 1998; 351: 9-13.

15. Johannes Steiner, Maya Guglin; BNP or NTpro-BNP? A clinician's perspective. International Journal of Cardiology 2008: 129: 5-14.

16. Duceppe E, Patel A, Chan MT, Berwanger O, Ackland G, Kavsak PA, et al. Preoperative N-terminal pro-B-type natriuretic peptide and cardiovascular events after noncardiac surgery: a cohort study. Ann Intern Med 2020; 172: 96-104. doi: 10.7326/M192501.

17. Jogia, PM, Kalkoff $M$, Sleigh JW, Bertinelli $A$, La Pine M, Richards AM, et al. NT-pro-BNP secretion and clinical endpoints in cardiac surgery intensive care patients. Anaesthesia and Intensive Care 2007: 35: 363-9. Doi: 10.1177/0310057X0703500307. 
18. Liu H, Wang C, Liu L, Zhuang $Y$, Yang $X$, Zhang $\mathrm{Y}$. Perioperative application of $\mathrm{N}$-terminal pro-brain natriuretic peptide in patients undergoing cardiac surgery. J Cardiothorac Surg 2013; 8: 1. doi:10.1186/1749-8090-8-1.

19. Schachner $\mathrm{T}$, Wiedemann $\mathrm{D}$, Fetz $\mathrm{H}$, Laufer $\mathrm{G}$, Kocher A, Bonaros N. Influence of preoperative serum $\mathrm{N}$-terminal pro-brain type natriuretic peptide on the postoperative outcome and survival rates of coronary artery bypass patients. Clinics 2010: 65: 1239-45.

20. Zheng $\mathrm{H}$, Li L, Liang H, Qu J, Li J, Cui Y, Chen X. Normalization of $\mathrm{N}$-terminal pro-B-type natriuretic peptide after cardiac surgery among children with tetralogy of Fallot. Am J Cardiol 2018; 122: 2125-30. doi: 10.1016/j.amjcard.2018.08.052.

21. Specifications Manual for Joint Commission National Quality Measures (v2018A) Discharges 07-01-18 (3Q18) through 12-31-18 (4Q18). Available from: https://manual.jointcommission.org/releases/TJC201 8A/DataElem0439.html

22. Ross RD, Bollinger RO, Pinsky WW. Grading the severity of congestive heart failure in infants. Pediatr Cardiol 1992; 13: 72-5.

23. KDIGO 2012 clinical practice guideline for evaluation and management of CKD. Kidney Int Suppl 2013: 3: 1-150. doi:10.1038/kisup.2012.72.

24. Nashef SA, Roques F, Sharples LD, Nilsson J, Smith $C$, Goldstone AR, et al. EuroSCORE II. Eur J Cardiothorac Surg 2012; 41: 734-45.

25. EuroSCORE II. Available from: URL: https://www.mdcalc.com/european-system-cardiacoperative-risk-evaluation-euroscore-ii

26. Januzzi JL, Camargo CA, Anwaruddin S, Baggish $A L$, Chen AA, Krauser DG, et al. The N-terminal ProBNP investigation of dyspnea in the emergency department (PRIDE) study. Am J Cardiol 2005; 95: 94854.

27. Lin C, Zeng X, Jiang S, Wu T, Wang J, Zhang J, et al. Role of the NT-pro-BNP level in the diagnosis of pediatric heart failure and investigation of novel combined diagnostic criteria. Exp Ther Med 2013; 6: 995-9. https://doi.org/10.3892/etm.2013.1250.

28. O'Donoghue $M$, Chen $A$, Baggish $A L$, Anwaruddin $S$, Krauser DG, Tung $R$, et al. The effects of ejection fraction on $\mathrm{N}$-terminal proBNP and BNP levels in patients with acute CHF: Analysis from the ProBNP Investigation of Dyspnea in the Emergency Department (PRIDE) Study. J Card Fail 2005: 11: S9S14. doi: 10.1016/j.cardfail.2005.04.011.
29. Wirth J, Buijsse B, di Giuseppe R, Fritsche A, Hense HW, Westphal S, et al. Relationship between Nterminal pro-brain natriuretic peptide, obesity and the risk of heart failure in middle-aged German adults. PLoS One 2014; 9: e113710. doi: 10.1371/journal.pone.0113710.

30. Krauser DG, Lloyd-Jones DM, Chae CU, Cameron R, Anwaruddin S, Baggish AL, et al. Effect of body mass index on natriuretic peptide levels in patients with acute congestive heart failure: a ProBNP Investigation of Dyspnea in the Emergency Department (PRIDE) substudy. Am Heart J 2005; 149: 744-50. doi: 10.1016/j.ahj.2004.07.010.

31. Lewis LK, Raudsepp SD, Prickett TCR, Yandle TG, Pemberton CJ, Richards AM. ProBNP processing is decreased by obesity in patients with heart failure. ATM 2020; 8: doi: 10.21037/atm.2019.12.81.

32. Su Y, Hou JY, Zhang YJ, Ma GG, Hao GW, Luo $\mathrm{JC}$, et al. Serum $\mathrm{N}$-terminal pro-B-type natriuretic peptide predicts mortality in cardiac surgery patients receiving renal replacement therapy. Front Med (Lausanne). 2020; 7: $153 . \quad$ doi: 10.3389/fmed.2020.00153.

33. Öztekin A, Memetoglu M, Kutlu R, Tekin A, Erbasan $O$, Arslan $U$, et al. The predictive value of preoperative serum NT-pro-BNP levels for the need for inotropic support in the postoperative period in patients undergoing coronary artery bypass grafting. Cardiovasc J 2017; 9: 90-6. doi: 10.3329/cardio.v9i2.32419.

34. Qu J, Liang H, Zhou N, Li L, Wang Y, Li J, et al. Perioperative NT-pro-BNP level: Potential prognostic markers in children undergoing congenital heart disease surgery. J Thorac Cardiovasc Surg 2017: 154: 631-40.

35. Brynildsen J, Petaja L, Pettila V,Nygard S, Vaara ST, Linko $R$ et al. The predictive value of NT-pro-BNP and hs-TnT for risk of death in cardiac surgical patients. Clin Biochem 2018; 53: 65-71.

36. Holm J, Vidlund $M$, Vanky $F$, Friberg $O$, Hakanson E, Walther S, et al. EuroSCORE II and Nterminal pro-B-type natriuretic peptide for risk evaluation: an observational longitudinal study in patients undergoing coronary artery bypass graft surgery. BJA: $\mathrm{Br} J$ Anaesth 2014; 113: 75-82. doi: 10.1093/bja/aeu

37. Cuthbertson BH, Croal BL, Rae D, Harrild K, Gibson $\mathrm{PH}$, Prescott GJ, et al. N-terminal pro-B-type natriuretic peptide concentrations and long -term outcome after cardiac surgery: a prospective cohort study. Br J Anaesth 2013; 110: 214-21. 